\title{
Synote: Designed for all Advanced Learning Technology for Disabled and Non-Disabled People
}

\author{
Mike Wald \\ School of Electronics and Computer Science, University of Southampton, United Kingdom \\ M.Wald@soton.ac.uk
}

\begin{abstract}
This paper describes the development and evaluation of Synote, a freely available accessible web based application that makes multimedia web resources (e.g. podcasts) easier to access, search, manage, and exploit for all learners, teachers and other users through the creation of accessible notes, bookmarks, tags, links, images and text captions synchronized to any part of the recording.
\end{abstract}

\section{Introduction}

Synote $^{i}$ has been designed to overcome the problem that while users can easily bookmark, search, link to, or tag the WHOLE of a podcast or video recording available on the web they cannot easily find, or associate their notes or resources with, PART of that recording. As an analogy, users would clearly find a text book difficult to use if it had no contents page, index or page numbers. Synote is freely available and uniquely can use speech recognition to automatically synchronise audio or video recordings with a transcript, slides and notes. Synote has been developed and evaluated with the involvement of users and support of JISC $^{\mathrm{ii}}$ and Net4Voice ${ }^{\mathrm{iii}}$. When the recording is replayed, currently spoken words are shown highlighted in the transcript. Selecting Synmarks (synchronised bookmarks, containing notes tags and links), transcript words or Slides/Images moves the recording to the corresponding times. The provision of text captions and images synchronized with audio and video enables all their communication qualities and strengths to be available as appropriate for different contexts, content, tasks, learning styles, learning preferences and learning differences. Text can reduce the memory demands of spoken language; speech can better express subtle emotions; while images can communicate moods, relationships and complex information holistically. Synote's synchronised transcripts enable the recordings to be searched while also helping support non native speakers and deaf and hearing impaired students understand the spoken text. The use of text descriptions and annotations of video or images help blind or visually impaired students understand the visual images.

\section{Speech Recognition Transcription}

Synote builds on many years of using of speech recognition for learning in collaboration with IBM and the international Liberated Learning Consortium (LLC). LLC formed in 1999 to trial real-time transcription for deaf students [1] using software to improve readability by automatically breaking up the text at pauses in the speech. All students liked using the corrected transcripts after the class but the accuracy from spontaneous speech was lower than from dictated speech. Further developments in collaboration with IBM included automatically synchronising audio, text and PowerPoint slides (which enabled recordings to be searched) and training the system using spontaneous speech rather than scripts. ViaScribe also provided a programming interface for the speech recognition output to be enhanced through interoperability with systems that; displayed the recognised text on personalized displays on networked laptops in ways users preferred (e.g. colour, font, size etc.); allowed the recognition errors to be correct in real time by one or more people; and merged the text output from any number of speakers each using ViaScribe [2]. IBM more recently developed the speaker independent 'Attila' system that can be hosted on the web, interfaced with other applications and used to transcribe, edit and display recordings created in a wide range of multimedia file formats. Synote adds user created notes and tags synchronised to the recordings, transcripts and slides provided by ViaScribe or Attila and the transcription process has been greatly 
simplified by integration of the IBM Hosted Transcription System with Synote.

\section{Classroom Use and Evaluation of Synote}

Synote is currently being used by teachers and students in the UK, Italy, Germany, Pakistan, Australia, US and Canada. Dr Wald has used Synote with over 40 recordings of his lectures with synchronised transcripts and slides for his teaching of approximately 200 students on 5 undergraduate and postgraduate modules in The University of Southampton's School of Electronics and Computer Science (ECS). There are over 500 recordings available on Synote (most with manually corrected synchronised transcripts, slides and annotations) for students to use for their learning. The provision of a verbatim synchronised transcript enables students to concentrate on learning and take only brief synchronised notes in Synote (e.g. 'revise this section for exam', 'I don't understand this fully' etc.). This feature is greatly valued by all students, not only by deaf students who need to lipread or watch a sign language interpreter and so can not take notes or dyslexic students or non-native speakers or others who find it difficult to take notes. The fact that Synote is used and valued by all students means that non native speakers and disabled students feel more included and do not have to use special technology. They have said that they do not like standing out from the crowd by having to walk to the front of the class to ask the teacher if they can record the lecture on their personal digital recorder. Also the quality of recording from a teacher's wireless headworn microphone is significantly better than from small digital recorders placed at the front of the class. Students did not like retyping handwritten notes they had taken in class into Synote after the recording had been uploaded and so Synote has recently been enhanced so that synchronised notes taken live in class on mobiles or laptops using Twitter ${ }^{\text {iv }}$ can be automatically uploaded into Synote.

Five classes with approximately 200 students were surveyed, with 101 students filling in questionnaires about their experience with Synote. The questionnaire results showed that Synote's support for a wide range of browsers was justified and appreciated and confirmed that Synote was easy to use as over $80 \%$ of the respondents didn't need to read or listen to the Synote guides and rated Synote as 4 or 5 on a 5 point scale of ease of use with the remaining percentage rating it as 3 . The design of Synote to provide synchronised slides, video, audio and transcripts was also appreciated with over $80 \%$ respondents rating
Synote as 4 or 5 for being useful overall as well as its slides, video and audio and transcript also each being useful with averages ratings of between 4.1 and 4.5. A slightly smaller percentage rated the Synmark option and the print out option as being useful with an average of 3.7 and 3.3 respectively on the 5 point scale used. One student commented that since there was no experience in using these facilities they were not being used but if Synote was used regularly then it would become second nature to use all these facilities. $97 \%$ of the students wanted all their lectures to be presented on Synote. Students stated that it was important that ALL lectures were recorded so they didn't find that the one lecture they missed hadn't been recorded. Of these 44 students $37 \%$ identified themselves as native speakers, $26 \%$ as fluent, $28 \%$ as having good language knowledge and $9 \%$ as having little language knowledge while $7 \%$ identified themselves as having hearing disabilities, $2 \%$ visual disabilities, $11 \%$ learning disabilities and $7 \%$ other disabilities Non native speakers in particularly commented how valuable Synote was for them as it was sometimes difficult to understand lecturers' speech and note-taking in a foreign language was very difficult for them. One commented that they could get words not understood in the transcript translated by Google.

\section{Conclusion and Future Work}

Synote has been shown to provide accessible and very well received enhancements to web based teaching and learning for all students and to integrate well with other applications including PowerPoint, Twitter and Speech Recognition Software.

\section{References}

[1] Leitch, D., MacMillan, T. Liberated Learning Initiative Innovative Technology and Inclusion: Current Issues and Future Directions for Liberated Learning Research. Saint Mary's University, Nova Scotia. 2003 http://www.liberatedlearning.com/

[2] Wald, M. and Bain, K. Enhancing the Usability of RealTime Speech Recognition Captioning through Personalised Displays and Real-Time Multiple Speaker Editing and Annotation. In Proceedings of HCI International 2007: 12th International Conference on Human-Computer Interaction, Beijing.

\footnotetext{
${ }^{\mathrm{i}}$ http://www.synote.org

ii $h t t p: / / w w w . j i s c . a c . u k$

iii $h$ ttp://spazivirtuali.unibo.it/net4voice/default.aspx

${ }^{\text {iv }}$ http://twitter.com/synote
} 